\title{
Differential effects of docosahexaenoic acid on preterm and term placental pro-oxidant/antioxidant balance
}

\author{
Michael J Stark ${ }^{1,2}$, Vicki L Clifton ${ }^{1}$ and Nicolette A Hodyl ${ }^{1}$ \\ ${ }^{1}$ The Robinson Institute, University of Adelaide, 77 King William Road, North Adelaide, South Australia 5006, \\ Australia and ${ }^{2}$ The Department of Perinatal Medicine, Women's and Children's Hospital, Adelaide, \\ South Australia, Australia
}

Correspondence should be addressed to M J Stark; Email: michael.stark@adelaide.edu.au

\begin{abstract}
Docosahexaenoic acid (DHA) supplementation in pregnancy may confer some clinical benefits; however, this compound can exert pro-oxidant effects. In this study, we investigated the effects of DHA on pro-oxidant/antioxidant balance in term and preterm placental explants, assessing oxidative stress marker concentrations, antioxidant capacity and pro-inflammatory cytokine production. Term $(n=8)$ and preterm $(n=9)$ placental explants were exposed to lipopolysaccharide (LPS, $1 \mathrm{ng} / \mathrm{ml})$, DHA $(1,10$ and $100 \mu \mathrm{M})$, and DHA and LPS simultaneously or pre-treated with DHA for $24 \mathrm{~h}$ prior to LPS treatment. The production of malondialdehyde (MDA, lipid peroxidation), 8-hydroxy-2-deoxy guanosine (8-OHdG, oxidative DNA damage) and pro-inflammatory cytokines (tumour necrosis factor $\alpha$ (TNF $\alpha$ ), interleukin 6 and interferon- $\gamma$ ) and total antioxidant capacity were measured. DHA at a concentration of $100 \mu \mathrm{M}$ induced oxidative stress in term placentas, while at all the three concentrations, it induced oxidative stress in preterm placentas. DHA and LPS resulted in reduced MDA levels in term $(P<0.005)$ and preterm $(P=0.004)$ placentas and reduced 8-OHdG levels in preterm placentas $(P=0.035)$. DHA pre-treatment, but not co-treatment with LPS, reduced 8-OHdG levels $(P<0.001)$ in term placentas. DHA increased antioxidant capacity only in term placentas $(P<\mathbf{0 . 0 0 1})$, with lower antioxidant capacity being observed overall in preterm placentas compared with term placentas $(P \leq \mathbf{0 . 0 0 1})$. In term placentas, but not in preterm ones, DHA co-treatment and pre-treatment reduced LPS-induced TNF $\alpha$ levels. The ability of DHA to alter placental pro-oxidant/antioxidant balance is dependent on the DHA concentration used and the gestational age of the placental tissue. DHA has a greater capacity to increase oxidative stress in preterm placentas, but it offers greater protection against inflammation-induced oxidative stress in term placentas. This appears to be a result of DHA altering placental antioxidant capacity. These data have implications for the timing and concentration of DHA supplementation in pregnancy.

Reproduction (2013) $146243-251$
\end{abstract}

\section{Introduction}

Fish oil supplementation is beneficial for the treatment of autoimmune, inflammatory and cardiovascular disorders, with its effects being attributed to the high content of the $n-3$ fatty acid docosahexaenoic acid (DHA, 22:6n-3). DHA modulates a number of cell functions and exerts a wide range of biological effects including alterations in endothelial function, vascular reactivity, leukocyte and macrophage function, cytokine production and reactive oxygen species (ROS) production (Gorjao et al. 2009). In pregnancy, dietary supplementation with $n-3$ fatty acids confers significant clinical benefits, reducing the incidence of spontaneous preterm birth (Olsen et al. 2000, Smuts et al. 2003, Makrides et al. 2010).

Pregnancy is characterised by a balance between the increased production of ROS and the antioxidant defences that protect the human placenta and fetus. The placenta is an important source of products of oxidative stress, such as lipid peroxides (Mutlu-Turkoglu et al. 1998). Theoretical concerns exist that dietary $n-3$ fatty acid supplementation may lead to increased placental oxidative stress (Shoji et al. 2009). Exposure of placental cells to low-dose $n-3$ fatty acids has been found to alleviate oxidative DNA damage, while higher doses have been reported to accelerate lipid peroxidation. However, evidence from a recent study in rats suggests a protective effect of $n-3$ fatty acid supplementation on the placenta and developing fetus, resulting in increased $n-3$ fatty acid composition of the placenta and increased placental and fetal weights compared with those in dams fed a control diet (Jones et al. 2013). These alterations were associated with a significant reduction in the levels of markers of placental oxidative stress (F2-isoprostanes), suggesting that $n-3$ fatty acid supplementation is not harmful to the developing fetus and, importantly, contributes to the maintenance of placental oxidative balance. Therefore, $n-3$ fatty acids may improve pregnancy outcomes by 
protecting against oxidative damage, which is elevated in conditions that complicate pregnancy (Fujimaki et al. 2011) and result in preterm birth (Myatt 2010).

The mechanisms underlying $n-3$ fatty acid-mediated effects on free radical homeostasis remain unclear, but they could involve alterations in ROS production (Fisher et al. 1990) and the expression and activity of antioxidant enzymes (Takahashi et al. 2002) or a reduction in pro-inflammatory mediator release that can lead to increased ROS production (Mori et al. 2003). Given that the rates of preterm birth are reduced with $n-3$ fatty acid supplementation, without an increase in the overall length of gestation period (Makrides et al. 2010), preterm placentas may be more sensitive to the beneficial effects of $n-3$ fatty acids than term placentas. Therefore, the objectives of this study were to investigate the effect of DHA on placental oxidative stress and determine whether DHA could attenuate placental oxidative stress induced by lipopolysaccharide (LPS) exposure in a human explant model, using term and preterm placentas. Placentas were obtained from nonlabour caesarean sections, as labour itself is known to increase oxidative stress (Hung et al. 2011). The concentrations of markers of placental antioxidant capacity and pro-inflammatory cytokine production were also measured to assess potential mechanistic pathways.

\section{Materials and methods}

The Central and Northern Area Health Service, Women's and Children's Health Network and University of Adelaide Human Research Ethics Committees approved the study. Pregnant women not using fish oil or DHA supplements were recruited, and they provided written informed consent prior to nonlabour caesarean section at term (37-41 weeks; $n=8)$ or preterm ( $<37$ weeks of gestation; $n=9$ ) at the Lyell McEwin Hospital and the Women's and Children's Hospital (Adelaide, South Australia). Birth weight, placental weight and fetal sex were determined at birth.

\section{Placental explant culture}

Placental tissue was collected and pooled from six sites across the maternal surface of the placenta. It was trimmed to remove major blood vessels and reduce decidual contamination and washed with saline to remove maternal blood. It was cut into $\sim 5 \mathrm{~mm}$ pieces, and 80-100 mg were placed in each well of a 24-well plate, with the exact weight in each well being recorded. The explants were incubated in $1 \mathrm{ml} \mathrm{CMRL-1066}$ medium (Gibco, Invitrogen Australia Pty Ltd.) supplemented with 5\% FCS (JRH Biosciences, Inc., Lenexa, KS, USA), $100 \mathrm{mg} / \mathrm{ml}$ gentamicin (Pfizer Pty Ltd.) and $100 \mathrm{ng} / \mathrm{ml}$ retinyl acetate (Sigma-Aldrich) and cultured for $24 \mathrm{~h}$ at $37^{\circ} \mathrm{C}$ and $5 \%$ $\mathrm{CO}_{2}$. Following the $24-\mathrm{h}$ incubation, the medium was removed and replaced with a serum-free medium and the treatments were commenced. Each treatment was performed in triplicate.

\section{Placental explant stimulation studies}

DHA was reconstituted in ethanol (UNIVAR Analytical Reagent, Tarren Point, NSW, Australia) and prepared at three concentrations (1, 10 and $100 \mu \mathrm{M})$ previously found to induce oxidative stress in BeWo cells (Shoji et al. 2009). Term placental explants were cultured with increasing doses of $\mathrm{DHA}$ to determine the concentration-dependent induction of oxidative stress. Explant culture conditions, including optimal incubation time periods ( 6 and $24 \mathrm{~h}$ ) and LPS concentration $(1 \mathrm{ng} / \mathrm{ml})$, were used, as described previously (Scott et al. 2011). Briefly, placental explants were treated with LPS (Sigma-Aldrich), DHA alone, or DHA and LPS simultaneously or pre-treated with DHA for $24 \mathrm{~h}$ prior to the addition of LPS. Control wells included those with no treatment compound (i.e. media only) or the DHA vehicle (ethanol). The final concentration of ethanol in the culture medium for any experimental condition was $<0.1 \%$. The supernatants were collected and stored at $-20{ }^{\circ} \mathrm{C}$ until analysis.

\section{Placental oxidative stress and antioxidant capacity}

The concentration of malondialdehyde (MDA), an advanced stable lipid peroxidation end product, was measured in explant supernatants using ELISA (OxiSelect, Cell Biolabs, Inc., San Diego, CA, USA), and it is expressed as picomoles per milligram placenta. The concentration of 8-hydroxy-2-deoxy guanosine $(8-\mathrm{OHdG})$, produced by the oxidative damage of DNA by ROS and reactive nitrogen species, was measured in explant supernatants using ELISA (Stressmarq Biosciences, Victoria, BC, Canada), and it is expressed as picograms per millilitre. The total antioxidant capacity (TAC) of explant supernatants was measured using the TAC assay kit (OxiSelect, Cell Biolabs, Inc.), with the results being expressed as $\mu \mathrm{M}$ copper reducing equivalents. The limits of detection were $1.87 \mathrm{pmol} / \mathrm{mg}$ (MDA), $10.3 \mathrm{pg} / \mathrm{ml}(8-\mathrm{OHdG})$ and $8.54 \mu \mathrm{M}$ copper reducing equivalents (TAC). All data were normalised for the weight of placental tissue in each well.

\section{Placental cytokine production}

The concentrations of pro-inflammatory cytokines (tumour necrosis factor $\alpha$ (TNF $\alpha$ ), interleukin 6 (IL6) and interferon- $\gamma$ $(\mathrm{IFN} \gamma)$ ) of explant supernatants were measured using ELISA (R\&D Systems, Inc., Minneapolis, MN, USA), and the results are expressed as picograms per millilitre. The limits of detection were $9.4 \mathrm{pg} / \mathrm{ml}$ (IL6) and $7.6 \mathrm{pg} / \mathrm{ml}$ (TNF $\alpha$ and IFN $\gamma$ ). The inter-assay coefficients of variation (CV) were 8.9\% (IL6), $11.1 \%(\mathrm{TNF} \alpha)$ and $9.2 \%(\mathrm{IFN} \gamma)$. All data were normalised for the weight of placental tissue in each well.

\section{Statistical analyses}

All data are expressed as medians (interquartile range), unless otherwise indicated. Statistical analyses were performed using SPSS v19.0 (SPSS). Differences between control, DHA and LPS conditions were tested using non-parametric Kruskal-Wallis test and Mann-Whitney $U$ tests for post hoc comparisons where indicated adopting a Bonferroni's correction. Term and 
preterm responses were compared using Mann-Whitney $U$ tests, adopting Bonferroni's corrections for multiple comparisons. In order to remove potential confounding by the underlying disease states, the analyses were repeated by removing the results obtained from placentas associated with pathophysiological pregnancies (including chorioamnionitis and gestational diabetes) to assess their contribution. A $P$ value $<0.05$ was considered statistically significant.

\section{Results}

\section{Clinical characteristics}

Maternal, neonatal and obstetric characteristics related to the placentas used in this study are presented in Table 1. There was no difference in maternal age, parity, gravidity, cigarette smoking rate or pre-existing hypertension between women who delivered at term and those who delivered at preterm (Table 1). The rates of obstetric complications were also equal between the preterm and term groups. As expected, gestational length was significantly shorter in the preterm group compared with that in the term group, as were birth weight, birth length and head circumference. Placental weight was also equal between the two groups; however, there was more variation in the preterm group compared with the term group, owing to the inclusion of a placenta from a twin delivery in this group. All placentas were collected from women undergoing non-labour caesarean sections, with indications for delivery including maternal health conditions (pre-eclampsia or chorioamnionitis), suspected slowing of fetal growth or suspected intrauterine growth restriction, or previous caesarean section. Maternal antenatal steroids were administered to three women in the preterm group and one woman in the term group, who had presented to the hospital earlier in gestation due to threatened preterm labour.

\section{Effect of DHA concentration on placental oxidative stress}

The levels of oxidative stress markers (MDA and 8-OHdG) produced by term placental explants did not differ between the vehicle and media conditions, at either 6 or $24 \mathrm{~h}$. The levels of MDA were significantly increased following incubation of placental explants with $100 \mu \mathrm{M}$ DHA for both $6 \mathrm{~h}\left(\chi^{2}=8.043, P=0.045\right)$ and $24 \mathrm{~h}\left(\chi^{2}=8.646, P=0.03\right)$ compared with those observed for both control conditions (media and vehicle) and at 1 and $10 \mu \mathrm{M}$ DHA concentrations (Fig. 1A). The MDA levels of placental explants exposed to the 1 and $10 \mu \mathrm{M}$ DHA concentrations did not differ significantly from each other or from those observed for either of the control conditions. The production of $8-\mathrm{OHdG}$ in placental explants was significantly increased at all the three concentrations of DHA compared with that observed for the control conditions at $6 \mathrm{~h}\left(\chi^{2}=21.231\right.$,
Table 1 Maternal, neonatal and obstetric characteristics according to preterm $(<37$ weeks $)$ and term delivery.

\begin{tabular}{|c|c|c|c|}
\hline & $\begin{array}{c}\text { Preterm } \\
(n=9)\end{array}$ & $\begin{array}{l}\text { Term } \\
(n=8)\end{array}$ & $\boldsymbol{P}$ \\
\hline \multicolumn{4}{|l|}{ Maternal characteristics } \\
\hline Maternal age (years) & $36(6)$ & $34(6)$ & NS \\
\hline Parity, median (min-max) & $1(0-5)$ & $1(0-4)$ & NS \\
\hline Gravidity, median (min-max) & $3(1-8)$ & $2(1-8)$ & NS \\
\hline Cigarette smoking, $n$ (\%) & $1(11)$ & $1(12)$ & NS \\
\hline Pre-existing hypertension, $n(\%)$ & $1(11)$ & 0 & NS \\
\hline \multicolumn{4}{|l|}{ Obstetric characteristics } \\
\hline Gestation period length (weeks) & $33(3)$ & $38(1)$ & 0.003 \\
\hline Gestational diabetes, $n(\%)$ & $2(22)$ & $1(12)$ & NS \\
\hline Pre-eclampsia, $n(\%)$ & $2(22)$ & 0 & NS \\
\hline PROM, $n(\%)$ & $1(11)$ & 0 & NS \\
\hline Antenatal steroid therapy, $n(\%)$ & $3(33)$ & $1(12)$ & NS \\
\hline Chorioamnionitis, $n(\%)$ & $1(11)$ & 0 & NS \\
\hline Placental weight & $522(209)$ & $654(95)$ & NS \\
\hline Twin pregnancy, $n(\%)$ & $1(11)$ & 0 & NS \\
\hline \multicolumn{4}{|l|}{ Neonatal characteristics } \\
\hline Male, $n(\%)$ & $5(55)$ & $6(75)$ & NS \\
\hline Birth weight (g) & $2119(750)$ & 3465 (328) & 0.002 \\
\hline Birth weight centile, mean (s.E.M.) & $42(15)$ & $45(12)$ & NS \\
\hline Birth length $(\mathrm{cm})$ & $43(4.5)$ & $50(1.5)$ & 0.003 \\
\hline Head circumference $(\mathrm{cm})$ & $31(3.3)$ & $35(0.6)$ & 0.008 \\
\hline SGA $(<10$ BWC $), n(\%)$ & $1(11)$ & 0 & NS \\
\hline Apgar 1 min, median (min-max) & $7(1-9)$ & $8(4-9)$ & NS \\
\hline Apgar 5 min, median (min-max) & $9(7-9)$ & $9(8-9)$ & NS \\
\hline
\end{tabular}

Data represent means (s.D.), unless otherwise mentioned. Means were compared using $t$-tests, medians were compared using Mann-Whitney $U$ tests and frequency data were analysed using a $\chi^{2}$ analysis. NS, not significant; PROM, premature rupture of membranes; SGA, small for gestational age; BWC, birth weight centile.

$P<0.001)$ and $24 \mathrm{~h}\left(\chi^{2}=19.354, P<0.001\right.$; Fig. $\left.1 \mathrm{~B}\right)$. No significant difference was observed at the three DHA concentrations. The time of incubation had no effect on the production of either MDA or $8-\mathrm{OHdG}$ at each concentration of DHA.

Given that the aim of this set of experiments was to identify the potential mechanisms responsible for the beneficial effects of DHA in pregnancy, we continued to carry out the experiments using only the lower two DHA concentrations that did not induce increased placental lipid peroxidation. In preterm placentas, DHA alone (both 1 and $10 \mu \mathrm{M}$ ) significantly increased both MDA (Fig. 2B) and 8-OHdG (Fig. 2D; $P=0.001$ in both instances) levels above the control levels (media and vehicle), an effect not observed in term placentas (Fig. 2A and C).

\section{Effect of DHA on LPS-induced oxidative stress and antioxidant capacity}

The time of incubation and concentration of DHA had no effect on the levels of any of the markers of oxidative stress or antioxidant capacity measured in each explant condition (Table 2). Therefore, the data are presented grouped across time and DHA concentration.

In term placentas, LPS induced a significant increase in MDA (Fig. $2 \mathrm{~A} ; \chi^{2}=30.903, P<0.01$ ) and $8-\mathrm{OHdG}$ 

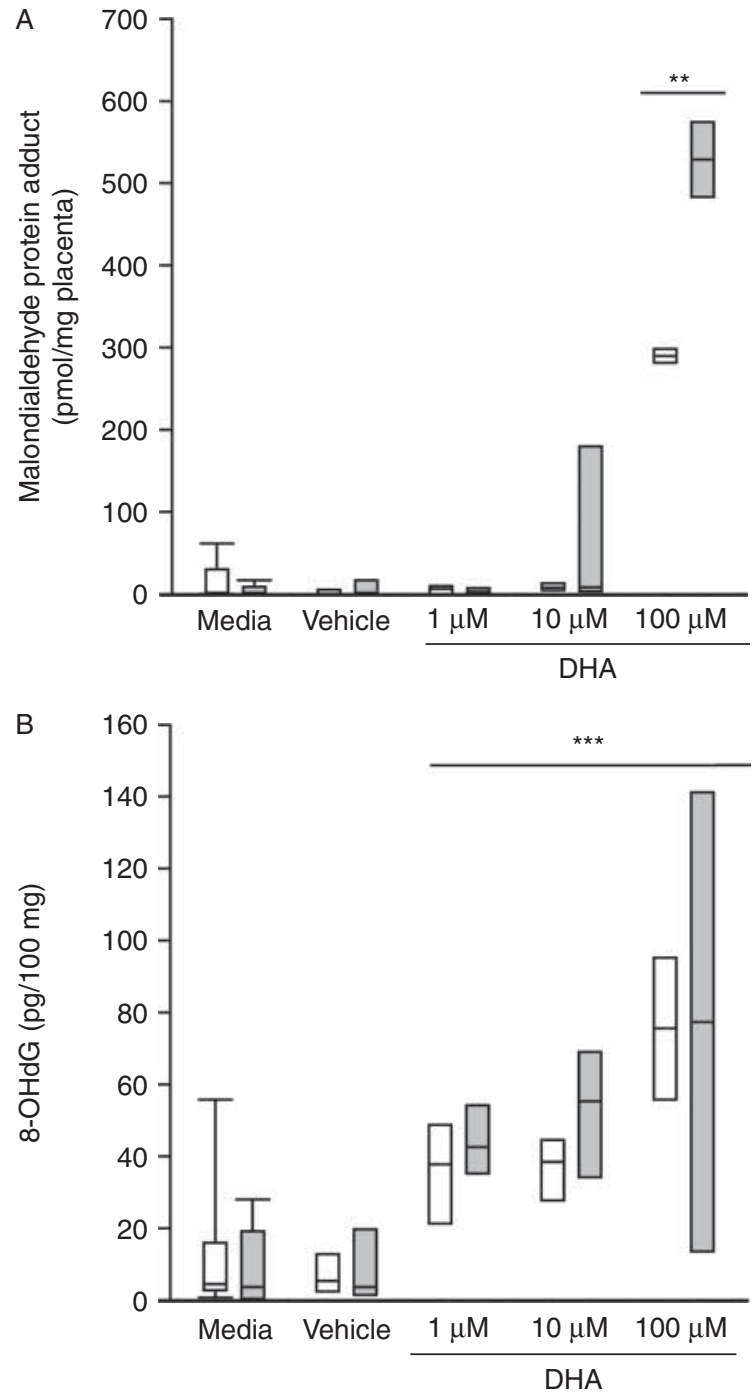

Figure 1 Effect of increasing concentrations of docosahexaenoic acid on malondialdehyde (A) and 8-hydroxy-2-deoxy guanosine (B) levels in term placental explants collected at $6 \mathrm{~h}$ (white boxes) and $24 \mathrm{~h}$ (grey boxes). ${ }^{* *} P<0.001$, DHA $100 \mu \mathrm{M}$ compared with all other conditions. *** $P<0.001$, all DHA concentrations compared with media and vehicle controls. Analyses were conducted using the Kruskal-Wallis test with Mann-Whitney post hoc analyses. Boxes and whiskers represent the interquartile range and $95 \% \mathrm{Cl}$, with bars representing the median values.

(Fig. 2C; $\left.\chi^{2}=74.09, P<0.001\right)$ levels and a significant decrease in TAC (Fig. 2E; $\chi^{2}=14.612, P=0.006$ ) compared with DHA alone or media/vehicle. The combination of LPS and DHA (either simultaneously or with 24-h DHA pre-treatment) resulted in significantly lower MDA levels compared with LPS treatment (Fig. 2A; $P<0.005)$, to a level similar to those observed in both DHA-alone and control conditions. The simultaneous exposure of term placental explants to DHA and LPS did not reduce the production of 8-OHdG compared with LPS exposure alone (Fig. 2C). However, DHA pre-treatment of term placental explants prior to LPS exposure significantly reduced the production of 8-OHdG compared with LPS exposure alone (Fig. 2C; $P<0.001)$. The TAC of term placentas was maintained at control levels following exposure to DHA, either alone or in combination with LPS (both with the co-treatment and pre-treatment exposures; Fig. 2E).

In preterm placentas, LPS induced a significant increase in MDA (Fig. $2 \mathrm{~B} ; \chi^{2}=42.332, P<0.001$ ) and 8-OHdG (Fig. 2D; $\chi^{2}=40.906, \quad P<0.001$ ) levels compared with DHA alone or media/vehicle. The levels of MDA were significantly increased during all exposures compared with the controls (Fig. 2B; $P<0.001$ in each case). DHA and LPS (co-treatment and DHA pretreatment) significantly reduced the production of MDA compared with LPS alone (Fig. 2B; $P=0.004$ and $P=0.035$ respectively). DHA and LPS (co-treatment and DHA pre-treatment) significantly reduced the production of 8-OHdG compared with LPS alone (Fig. 2D; $P=0.035$ and $P=0.037$ respectively). The production of $8-\mathrm{OHdG}$ was significantly increased during all exposures compared with the controls (Fig. 2D; $P<0.001$ in each case). In preterm placentas, there was no change in TAC with any treatment (Fig. 2F).

The term and preterm placentas produced equivalent levels of MDA in response to LPS; however, MDA levels were significantly lower in preterm placentas compared with term placentas following exposure to DHA alone or in combination with LPS (co-treatment and pretreatment; $P \leq 0.001$ in all cases). Similarly, preterm placentas produced significantly less $8-\mathrm{OHdG}$ than term placentas following exposure to DHA, LPS, or the combination of DHA and LPS ( $P \leq 0.001$ in all cases). TAC was significantly lower in preterm placentas compared with term placentas in each condition $(P \leq 0.001$ in all cases).

\section{Effect of DHA on LPS-induced pro-inflammatory cytokine production}

The time of incubation and concentration of DHA had no effect on cytokine production, and the data were, therefore, collapsed across these variables. The concentrations of IL6 and IFN $\gamma$ did not differ significantly between each DHA and LPS condition for either term or preterm placentas (Table 3). The production of TNF $\alpha$ was significantly increased following exposure of both preterm and term placentas to LPS (preterm $P=0.013$; term $P=0.009$ ). In term placentas, both DHA co-treatment and pre-treatment significantly reduced TNF $\alpha$ levels following LPS exposure to levels observed in the controls. However, in preterm placentas, TNF $\alpha$ levels remained significantly elevated in the presence of LPS and DHA, at levels equivalent to those induced by LPS alone (Table 3). The production of cytokines in term and preterm placental explants was similar, with the exception of that of IL6 and TNF $\alpha$ in the media/vehicle 

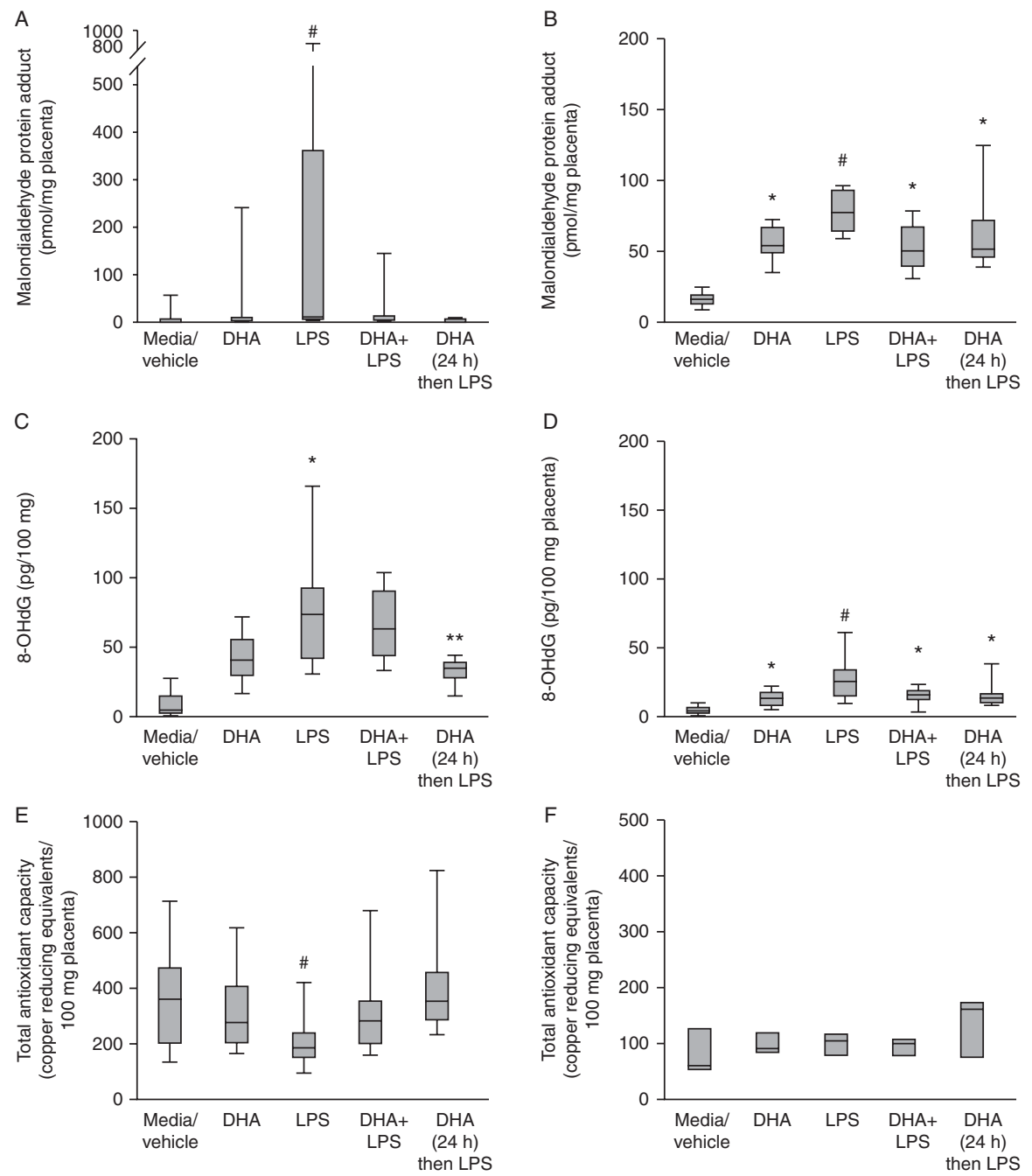

Figure 2 Effect of DHA alone ( 1 and $10 \mu \mathrm{M})$, LPS $(1 \mathrm{ng} / \mathrm{ml})$ alone, DHA and LPS co-treatment or DHA pre-treatment $24 \mathrm{~h}$ before LPS treatment on the production of malondialdehyde in term $(\mathrm{A})$ and preterm (B) placentas, 8-hydroxy-2-deoxy-guanosine in term $(\mathrm{C})$ and preterm $(\mathrm{D})$ placentas, and total antioxidant capacity in term (E) and preterm (F) placentas. ${ }^{\sharp} P<0.005$ compared with all other conditions; ${ }^{*} P<0.001$ compared with media/vehicle; and ** $P<0.015$ compared with DHA, LPS and DHA + LPS. Analyses were conducted using the Kruskal-Wallis test with post hoc comparisons. Boxes and whiskers represent the interquartile range and $95 \% \mathrm{Cl}$, with bars representing the median values.

conditions where cytokine levels were significantly higher in the preterm explants $(P<0.001$ in each case). The presence of pregnancy complications (gestational diabetes, pre-eclampsia or chorioamnionitis) did not affect placental responses to DHA or LPS.

\section{Discussion}

This study has demonstrated that the ability of DHA to alter the pro-oxidant/antioxidant balance in the placenta is dependent on the DHA concentration used and the gestational age of the placental tissue. Specifically, and

Table $2 \mathrm{MDA}$ and 8-OHdG concentrations, TAC and cytokine concentrations in the supernatants of placental explants exposed to DHA at two concentrations ( 1 and $10 \mu \mathrm{M}$ ) for 6 and $24 \mathrm{~h}$.

\begin{tabular}{|c|c|c|c|c|c|c|c|c|}
\hline & \multicolumn{3}{|c|}{$6 \mathrm{~h}$} & \multicolumn{3}{|c|}{$24 \mathrm{~h}$} & \multirow{2}{*}{$\begin{array}{c}\boldsymbol{P}^{\mathrm{a}} \\
6 \text { vs } 24 \\
\end{array}$} & \multirow{2}{*}{$\begin{array}{c}\boldsymbol{P}^{\mathrm{b}} \\
6 \text { vs } 24 \\
\end{array}$} \\
\hline & $1 \mu \mathrm{M} \mathrm{DHA}$ & $10 \mu \mathrm{M}$ DHA & $P$ & $1 \mu \mathrm{M} \mathrm{DHA}$ & $10 \mu \mathrm{M}$ DHA & $P$ & & \\
\hline MDA (pmol) & $5.9(0-8.5)$ & $6.8(1.9-10.9)$ & 0.4 & $3.1(0-6.4)$ & $6.7(1.3-15.1)$ & 0.1 & 0.4 & 0.9 \\
\hline 8-OHdG (pg/ml) & $39.7(32.8-63.0)$ & $40.6(28.5-56.4)$ & 0.6 & $39.4(33.8-49.4)$ & $45.0(31.4-66.2)$ & 0.5 & 0.6 & 0.6 \\
\hline $\begin{array}{l}\text { TAC ( } \mu \mathrm{M} \text { copper } \\
\text { reducing } \\
\text { equivalents) }\end{array}$ & $280.4(219.1-364.8)$ & $302.9(254.9-391.2)$ & 0.7 & $312.0(285.7-546.3)$ & $278.6(194.8-432.3)$ & 0.2 & 0.2 & 0.9 \\
\hline $\mathrm{TNF} \alpha(\mathrm{pg} / \mathrm{ml})$ & $51.4(0-895)$ & $480(0-1229)$ & 0.2 & $49(4-1090)$ & $371(5-1264)$ & 0.5 & 0.6 & 0.9 \\
\hline IL6 (pg/ml) & $1702(4-10650)$ & $1621(264-3341)$ & 0.9 & 79 (10-12 393) & $2680(420-4294)$ & 0.2 & 0.6 & 0.3 \\
\hline $\mathrm{IFN} \gamma(\mathrm{pg} / \mathrm{ml})$ & $0(0-48)$ & $13(0-49)$ & 0.3 & $98(24-450)$ & $71(11-130)$ & 0.6 & 0.002 & 0.001 \\
\hline
\end{tabular}

Data are presented as medians (interquartile range). $P$ values represent significance level of comparison using the Mann-Whitney $U$ non-parametric test. MDA, malondialdehyde; 8-OHdG, 8-hydroxy-2-deoxy guanosine; TAC, total antioxidant capacity.

${ }^{\mathrm{a}} 1 \mu \mathrm{M}$ DHA. ${ }^{\mathrm{b}} 10 \mu \mathrm{M}$ DHA. 
Table 3 Concentrations of TNF $\alpha$, IL6 and IFN $\gamma$ in the supernatants of preterm and term placental explants exposed to media/vehicle, DHA, LPS or DHA + LPS co-treatment and 24-h DHA pre-treatment followed by LPS treatment.

\begin{tabular}{|c|c|c|c|c|c|c|}
\hline & Media/vehicle & DHA & LPS & LPS + DHA & DHA $(24 \mathrm{~h})$ and then LPS & $\boldsymbol{P}^{*}$ \\
\hline \multicolumn{7}{|l|}{$\mathrm{TNF} \alpha$} \\
\hline Preterm & $65(0-1054)$ & $40(0-480)$ & $951(32-1935)^{+}$ & $962(652-1300)^{+}$ & $1081(689-1370)^{+}$ & 0.013 \\
\hline Term & $0(0-3)$ & $0(0-22)$ & $998(0-1336)^{+}$ & $369(0-1308)$ & $642(0-1117)$ & 0.009 \\
\hline \multicolumn{7}{|l|}{ IL6 } \\
\hline Preterm & $1964(257-2725)$ & $1191(492-3340)$ & $2095(881-3182)$ & $1918(1558-2762)$ & $2536(1557-3288)$ & 0.42 \\
\hline Term & $178(5-648)$ & $462(11-5612)$ & $4540(0-11424)$ & $3711(20-11302)$ & $3463(25-11070)$ & 0.11 \\
\hline \multicolumn{7}{|l|}{$\mathrm{IFN} \gamma$} \\
\hline Preterm & $10(0-71)$ & $4(0-60)$ & $4(0-25)$ & $17(0-65)$ & $26(0-92)$ & 0.14 \\
\hline Term & $0(0-14)$ & $3(0-52)$ & $93(0-143)$ & $0(0-62)$ & $31(0-83)$ & 0.051 \\
\hline
\end{tabular}

Data are presented as medians (interquartile range). ${ }^{*} P$, significance level of the Kruskal-Wallis non-parametric test, comparing across conditions. ${ }^{+} P<0.03$ compared with media/vehicle and DHA-alone groups (post hoc Mann-Whitney $U$ test).

as observed in a previous study (Shoji et al. 2009), a high concentration of DHA $(100 \mu \mathrm{M})$ induced placental oxidative stress (lipid peroxidation and oxidative DNA damage) in term placental tissue, while lower concentrations (both 1 and $10 \mu \mathrm{M}$ DHA) did not increase placental lipid peroxidation, yet still resulted in a degree of oxidative DNA damage. In preterm placental tissue, the lower doses of DHA resulted in increased lipid peroxidation and oxidative DNA damage. In response to an inflammatory challenge, the lower doses of DHA were able to ameliorate both LPS-induced oxidative stress and TNF $\alpha$ production and restore antioxidant capacity in term placentas. Conversely, in preterm placentas, DHA did not reduce LPS-induced proinflammatory cytokine production or increase placental antioxidant capacity, although a reduction in LPSinduced oxidative stress was observed. However, it should be noted that in preterm placentas DHA only reduced LPS-induced lipid peroxidation to the level induced by DHA alone, while in term placentas, lipid peroxidation was reduced further to control levels. Together, these data suggest that DHA has a greater capacity to increase oxidative stress in preterm placentas compared with term placentas, suggesting that preterm placentas are more susceptible to oxidative damage as a result of limited placental antioxidant capacity. When exposed to inflammation, DHA appears to protect against oxidative damage to a greater degree in term placentas compared with preterm placentas, through an increase in antioxidant capacity and a reduction in proinflammatory cytokine production. The reduction in LPSinduced oxidative stress by DHA in preterm placentas does not appear to be a result of increased antioxidant capacity or a reduction in pro-inflammatory cytokine production. Further research is warranted to elucidate the mechanism contributing to decreased oxidative stress in the context of preterm delivery.

The suppression of pro-inflammatory cytokine production following DHA exposure has been observed previously in other tissues. For example, exposure of human THP-1 monocyte-derived macrophages to DHA (25 and $100 \mu \mathrm{M}$ ) prior to LPS stimulation reduced both
mRNA and protein expression of the pro-inflammatory cytokines TNF (TNF $\alpha$ ), IL6 and IL1B (IL1 $\beta$ ) and prevented

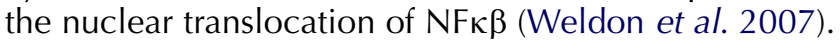
In the present study, we observed a similar effect of DHA treatment in term placentas, with DHA suppressing LPS-induced TNF $\alpha$ production back to basal levels. In preterm placentas, however, no such inhibition was observed with either DHA pre-treatment or DHA co-treatment. This may be due to the differential effects of DHA on term and preterm placentas, via actions on surface or intracellular receptors, such as the peroxisome proliferator-activated receptors (PPARs). PPARs are important transcription factors that when activated by $n-3$ fatty acids modulate inflammatory responses (Blanquart et al. 2003) including fetoplacental inflammation (Lappas et al. 2006). Placental PPARr is particularly notable because it has strong antiinflammatory properties, including inhibition of pro-inflammatory cytokine gene transcription (Lappas et al. 2002). Previous studies assessing the expression of placental PPARs (collected following vaginal delivery) have indicated that preterm ( 34 weeks of gestation) and term placentas have equivalent PPARA (PPAR $\alpha$ ) mRNA and protein levels; however, protein expression of PPARD (PPAR $\delta$ ) and PPARG (PPAR $\gamma$ ) is significantly higher in preterm placentas compared with term placentas (Holdsworth-Carson et al. 2009). However, DNA binding of all the PPAR isoforms did not differ between the preterm and term tissues (HoldsworthCarson et al. 2009), suggesting an equal functional capacity of PPARs across late gestation. However, labour is associated with a reduction in PPAR $\gamma$ levels (Dunn-Albanese et al. 2004). The effects of labour on preterm placental PPAR expression and function are currently unknown, but may explain the observed differences between term and preterm responses to DHA in the present study, given that we collected placentas from women undergoing non-labour caesarean sections. The reduced levels or activity of PPAR $\gamma$ in the preterm placentas compared with term placentas may contribute to the current findings and should be confirmed in future studies. 
DHA can also directly mediate cellular functions by altering cell membrane phospholipid composition. DHA supplementation, in both human and animal studies of pregnancy, increases DHA content of maternal and fetal plasma lipids and erythrocytes as well as placenta and fetal liver (Dunstan et al. 2004, Krauss-Etschmann et al. 2007, Jones et al. 2013). The phospholipid composition of the cell membrane is an important determinant of membrane protein function, membrane fluidity and lipid raft formation (for a review, see Calder (2012)). Lipid rafts are membrane microdomains, composed of cholesterol, sphingolipids and glycolipids, which function as a platform for the co-localisation of receptor-mediated signalling components, including the LPS receptor TLR4 (Wong et al. 2009). Because DHA has low affinity for cholesterol, it is not incorporated directly into lipid rafts; however, its incorporation into membrane non-raft regions appears to affect lipid raft formation, with downstream effects on TLR4 signal transduction (Wong et al. 2009, Kim et al. 2013). This dysregulation of lipid raft function by DHA may have contributed to the attenuation of LPS-induced cytokine production observed in the present study in the term placental explants. The lack of DHA-induced inhibition of cytokine responses in preterm placentas, despite an equivalent and robust response to LPS alone, may suggest that preterm placentas have a reduced ability to incorporate DHA into the placental membranes compared with term placentas. This was not assessed in the present study or previously in the literature, but it may provide critical information explaining this differential gestation-specific response.

In the present study, the TAC of preterm placentas was markedly lower than that of term placentas in all conditions, including baseline (control) levels. This is consistent with the known developmental pattern of enzymatic antioxidant expression and activity and the availability of non-enzymatic antioxidants across gestation. The activity of the primary enzymatic antioxidants (catalase, superoxide dismutase and glutathione reductase) increases over pregnancy, while that of placental glutathione peroxidase remains unchanged (Qanungo \& Mukherjea 2000). Conversely, serum or plasma levels of non-enzymatic antioxidants, including vitamin A (De Vriese et al. 2001) and $\beta$-carotene (Oostenbrug et al. 1998), decrease throughout pregnancy, while levels of $\alpha$-tocopherol increase (Oostenbrug et al. 1998, De Vriese et al. 2001). Together these gestational changes in antioxidant capacity appear to place the preterm newborn at an increased risk of ROSinduced injury (Shah \& Shah 2009). DHA has previously been shown to enhance glutathione reductase levels in response to oxidative stress in human fibroblast cells (Arab et al. 2006). This may have contributed to the reduction in LPS-induced oxidative stress and increase in antioxidant capacity observed with DHA treatment in the term placental tissue in the present study. As DHA did not enhance the antioxidant capacity of preterm placentas, this mechanism may be limited by the inherently low expression of antioxidant enzymes earlier in gestation.

Human studies indicate that dietary supplementation with $n$ - 3 fatty acids (fish oil) in pregnancy does not appear to increase the levels of markers of oxidative stress in maternal urine (Shoji et al. 2006), but that it can reduce cord plasma F2-isoprostane levels in the infant (Barden et al. 2004). This supplementation is associated with an increase in the length of gestation period and a reduced risk of preterm birth (Olsen et al. 2000, Smuts et al. 2003, Szajewska et al. 2006, Horvath et al. 2007, Makrides et al. 2010), although the majority of studies have been limited by small sample sizes. With oxidative stress being widely implicated in pathophysiological pregnancy, attention has been focused on the potential benefits of other antioxidant dietary interventions, such as vitamins $C$ and $E$ and selenium. Vitamin $C$ and $E$ supplementation reduces the levels of circulating markers of endothelial activation and lipid peroxidation (Chappell et al. 2002); however, meta-analyses have failed to demonstrate that this provides any clinical benefits (Rumbold \& Crowther 2005a, 2005b, Polyzos et al. 2007, Roberts etal.2010) and may even increase the rates of adverse pregnancy outcomes (Poston et al. 2006, Klemmensen et al. 2009). Clinical studies of selenium supplementation, an important component of the antioxidant glutathione peroxidase, in pregnancy are still awaited.

Underlying maternal disease states are associated with increased oxidative stress and inflammation. As such, we collected placentas from pregnancy cases associated with gestational diabetes, pre-eclampsia and chorioamnionitis and repeated the analysis to ensure that our findings were not influenced by these conditions. However, the results remained unchanged, with DHA reducing oxidative stress in LPS-stimulated term and preterm tissues and increasing antioxidant capacity only in term placentas. This suggests that the ability of DHA to influence pro-oxidant/antioxidant balance is dependent on gestational age, and this is unaffected by the presence of these conditions. However, our sample size for this subanalysis was small, and largescale studies should address these questions further. The large variability observed in placental responses to stimulation observed in this study is not uncommon in studies of placentas. It was not due to the cases associated with maternal disease, and indeed larger individual variations in placental responses were observed in term tissue compared with preterm tissue, where the incidence of maternal disease is less common. Large individual placental responses to stimulation are well documented in the literature, where inter-individual coefficients of variation have been reported up to $86 \%$ (Turner et al. 2006, Scott et al. 2011). This is a limitation of studying human placental samples. Many factors may contribute to these differences, including genotype, single-nucleotide polymorphisms in promoter regions of 
genes encoding cytokines (Hollegaard \& Bidwell 2006) and fetal sex (Scott et al. 2011).

\section{Summary}

Oxidative damage is elevated in conditions that complicate pregnancy and result in preterm birth (Myatt 2010), with excessive ROS production playing a significant role in the development of oxygen radical diseases of the newborn (Auten \& Davis 2009). Recent clinical studies of maternal dietary supplementation with fish oil or other DHA-containing supplements suggest that some clinical benefits exist, such as a reduction in preterm birth rates; however, this compound is well known to exert pro-oxidant effects. In this study, we demonstrated that the potential for DHA to induce placental oxidative stress was enhanced in preterm placentas and with high doses of DHA. Lower DHA doses were sufficient to inhibit LPS-induced oxidative stress and pro-inflammatory cytokine production and increased the antioxidant capacity of term placentas. However, DHA was unable to improve antioxidant capacity or inhibit pro-inflammatory cytokine production in preterm placentas. The reason for this effect is currently unknown, but it may be secondary to reduced DHA incorporation into preterm placentas. These findings have clear implications for DHA supplementation during pregnancy, with differential benefits and harm implicated according to the timing of supplementation and concentration of DHA consumed. Further benefits from DHA supplementation may be gained once the optimal concentration and timing of supplementation are established.

\section{Declaration of interest}

The authors declare that there is no conflict of interest that could be perceived as prejudicing the impartiality of the research reported.

\section{Funding}

This work was supported by Channel 7 Children's Research Foundation, Adelaide, and the Thrasher Research Foundation (award 9118). Salary support was provided through the National Health and Medical Research Council Fellowships (ID 565512 to M J Stark, ID 510703 to V L Clifton, and ID 1016379 to N A Hodyl).

\section{References}

Arab K, Rossary A, Flourie F, Tourneur Y \& Steghens JP 2006 Docosahexaenoic acid enhances the antioxidant response of human fibroblasts by upregulating $\gamma$-glutamyl-cysteinyl ligase and glutathione reductase. British Journal of Nutrition 95 18-26. (doi:10.1079/ BJN20051626)
Auten RL \& Davis JM 2009 Oxygen toxicity and reactive oxygen species: the devil is in the details. Pediatric Research 66 121-127. (doi:10.1203/ PDR.0b013e3181a9eafb)

Barden AE, Mori TA, Dunstan JA, Taylor AL, Thornton CA, Croft KD, Beilin LJ \& Prescott SL 2004 Fish oil supplementation in pregnancy lowers F2-isoprostanes in neonates at high risk of atopy. Free Radical Research 38 233-239. (doi:10.1080/10715760310001656722)

Blanquart C, Barbier O, Fruchart JC, Staels B \& Glineur C 2003 Peroxisome proliferator-activated receptors: regulation of transcriptional activities and roles in inflammation. Journal of Steroid Biochemistry and Molecular Biology 85 267-273. (doi:10.1016/S0960-0760(03)00214-0)

Calder PC 2012 Mechanisms of action of (n-3) fatty acids. Journal of Nutrition 142 592S-599S. (doi:10.3945/jn.111.155259)

Chappell LC, Seed PT, Kelly FJ, Briley A, Hunt BJ, Charnock-Jones DS, Mallet A \& Poston L 2002 Vitamin C and E supplementation in women at risk of preeclampsia is associated with changes in indices of oxidative stress and placental function. American Journal of Obstetrics and Gynecology 187 777-784. (doi:10.1067/mob.2002.125735)

De Vriese SR, Dhont M \& Christophe AB 2001 Oxidative stability of low density lipoproteins and vitamin $\mathrm{E}$ levels increase in maternal blood during normal pregnancy. Lipids 36 361-366. (doi:10.1007/s11745-0010728-2)

Dunn-Albanese LR, Ackerman WE, Xie Y, lams JD \& Kniss DA 2004 Reciprocal expression of peroxisome proliferator-activated receptor- $\gamma$ and cyclooxygenase-2 in human term parturition. American Journal of Obstetrics and Gynecology 190 809-816. (doi:10.1016/j. ajog.2003.09.052)

Dunstan JA, Mori TA, Barden A, Beilin LJ, Holt PG, Calder PC, Taylor AL \& Prescott SL 2004 Effects of n-3 polyunsaturated fatty acid supplementation in pregnancy on maternal and fetal erythrocyte fatty acid composition. European Journal of Clinical Nutrition 58 429-437. (doi:10.1038/sj.ejcn.1601825)

Fisher M, Levine PH, Weiner BH, Johnson MH, Doyle EM, Ellis PA \& Hoogasian JJ 1990 Dietary n-3 fatty acid supplementation reduces superoxide production and chemiluminescence in a monocyte-enriched preparation of leukocytes. American Journal of Clinical Nutrition $\mathbf{5 1}$ 804-808.

Fujimaki A, Watanabe K, Mori T, Kimura C, Shinohara K \& Wakatsuki A 2011 Placental oxidative DNA damage and its repair in preeclamptic women with fetal growth restriction. Placenta 32 367-372. (doi:10.1016/j.placenta.2011.02.004)

Gorjao R, Azevedo-Martins AK, Rodrigues HG, Abdulkader F, ArcisioMiranda M, Procopio J \& Curi R 2009 Comparative effects of DHA and EPA on cell function. Pharmacology \& Therapeutics 122 56-64. (doi:10.1016/j.pharmthera.2009.01.004)

Holdsworth-Carson SJ, Permezel M, Rice GE \& Lappas M 2009 Preterm and infection-driven preterm labor: the role of peroxisome proliferatoractivated receptors and retinoid $X$ receptor. Reproduction 137 1007-1015. (doi:10.1530/REP-08-0496)

Hollegaard MV \& Bidwell JL 2006 Cytokine gene polymorphism in human disease: on-line databases, Supplement 3. Genes and Immunity 7 269-276. (doi:10.1038/sj.gene.6364301)

Horvath A, Koletzko B \& Szajewska H 2007 Effect of supplementation of women in high-risk pregnancies with long-chain polyunsaturated fatty acids on pregnancy outcomes and growth measures at birth: a metaanalysis of randomized controlled trials. British Journal of Nutrition 98 253-259. (doi:10.1017/S0007114507709078)

Hung TH, Chen SF, Hsieh TT, Lo LM, Li MJ \& Yeh YL 2011 The associations between labor and delivery mode and maternal and placental oxidative stress. Reproductive Toxicology 31 144-150. (doi:10.1016/j.reprotox. 2010.11.009)

Jones ML, Mark PJ, Mori TA, Keelan JA \& Waddell BJ 2013 Maternal dietary omega-3 fatty acid supplementation reduces placental oxidative stress and increases fetal and placental growth in the rat. Biology of Reproduction 88 37. (doi:10.1095/biolreprod.112.103754)

Kim K, Jung N, Lee K, Choi J, Kim S, Jun J, Kim E \& Kim D 2013 Dietary omega-3 polyunsaturated fatty acids attenuate hepatic ischemia/ reperfusion injury in rats by modulating toll-like receptor recruitment into lipid rafts. Clinical Nutrition [in press]. (doi:10.1016/j.clnu.2012. 11.026)

Klemmensen A, Tabor A, Osterdal ML, Knudsen VK, Halldorsson TI, Mikkelsen TB \& Olsen SF 2009 Intake of vitamin C and E in pregnancy 
and risk of pre-eclampsia: prospective study among 57346 women. BJOG: an International Journal of Obstetrics and Gynaecology 116 964-974. (doi:10.1111/j.1471-0528.2009.02150.x)

Krauss-Etschmann S, Shadid R, Campoy C, Hoster E, Demmelmair H, Jimenez M, Gil A, Rivero M, Veszpremi B, Decsi T et al. 2007 Effects of fish-oil and folate supplementation of pregnant women on maternal and fetal plasma concentrations of docosahexaenoic acid and eicosapentaenoic acid: a European randomized multicenter trial. American Journal of Clinical Nutrition 85 1392-1400.

Lappas M, Permezel M \& Rice GE 2002 Regulation of pro-inflammatory cytokines in human gestational tissues by peroxisome proliferatoractivated receptor $\gamma$ : effect of 15 -deoxy- $\Delta(12,14)-P G J(2)$. Journal of Clinical Endocrinology and Metabolism 87 4667-4672. (doi:10.1210/jc. 2002-020613)

Lappas M, Permezel M \& Rice GE 2006 15-Deoxy- $\Delta^{12,14}$-prostaglandin $J_{2}$ and troglitazone regulation of the release of phospholipid metabolites, inflammatory cytokines and proteases from human gestational tissues. Placenta 27 1060-1072. (doi:10.1016/j.placenta.2005.11.009)

Makrides M, Gibson RA, McPhee AJ, Yelland L, Quinlivan J, Ryan P \& DOMinO Investigative Team 2010 Effect of DHA supplementation during pregnancy on maternal depression and neurodevelopment of young children: a randomized controlled trial. Journal of the American Medical Association 304 1675-1683. (doi:10.1001/jama.2010.1507)

Mori TA, Woodman RJ, Burke V, Puddey IB, Croft KD \& Beilin LJ 2003 Effect of eicosapentaenoic acid and docosahexaenoic acid on oxidative stress and inflammatory markers in treated-hypertensive type 2 diabetic subjects. Free Radical Biology \& Medicine 35 772-781. (doi:10.1016/ S0891-5849(03)00407-6)

Mutlu-Turkoglu U, Ademoglu E, Ibrahimoglu L, Aykac-Toker G \& Uysal M 1998 Imbalance between lipid peroxidation and antioxidant status in preeclampsia. Gynecologic and Obstetric Investigation 46 37-40. (doi:10.1159/000009994)

Myatt L 2010 Review: Reactive oxygen and nitrogen species and functional adaptation of the placenta. Placenta 31 (Suppl) S66-S69. (doi:10.1016/ j.placenta.2009.12.021)

Olsen SF, Secher NJ, Tabor A, Weber T, Walker JJ \& Gluud C 2000 Randomised clinical trials of fish oil supplementation in high risk pregnancies. Fish Oil Trials In Pregnancy (FOTIP) Team. BJOG: an International Journal of Obstetrics and Gynaecology 107 382-395. (doi:10.1111/j.1471-0528.2000.tb13235.x)

Oostenbrug GS, Mensink RP, AI MD, van Houwelingen AC \& Hornstra G 1998 Maternal and neonatal plasma antioxidant levels in normal pregnancy, and the relationship with fatty acid unsaturation. British Journal of Nutrition 80 67-73. (doi:10.1017/S0007114598001780)

Polyzos NP, Mauri D, Tsappi M, Tzioras S, Kamposioras K, Cortinovis I \& Casazza G 2007 Combined vitamin C and E supplementation during pregnancy for preeclampsia prevention: a systematic review. Obstetrical \& Gynecological Survey 62 202-206. (doi:10.1097/01.ogx.0000 256787.04807.da)

Poston L, Briley AL, Seed PT, Kelly FJ, Shennan AH \& C Vitamins in Pre-eclampsia Trial 2006 Vitamin C and vitamin E in pregnant women at risk for pre-eclampsia (VIP trial): randomised placebo-controlled trial. Lancet 367 1145-1154. (doi:10.1016/S0140-6736(06)68433-X)

Qanungo S \& Mukherjea M 2000 Ontogenic profile of some antioxidants and lipid peroxidation in human placental and fetal tissues. Molecular and Cellular Biochemistry 215 11-19. (doi:10.1023/A:1026511420505)

Roberts JM, Myatt L, Spong CY, Thom EA, Hauth JC, Leveno KJ, Pearson GD, Wapner RJ, Varner MW, Thorp JM Jr et al. 2010 Vitamins
$\mathrm{C}$ and $\mathrm{E}$ to prevent complications of pregnancy-associated hypertension. New England Journal of Medicine 362 1282-1291. (doi:10.1056/ NEJMoa0908056)

Rumbold A \& Crowther CA 2005a Vitamin C supplementation in pregnancy. Cochrane Database of Systematic Reviews. CD004072. (doi:10.1002/14651858.CD004072.pub2)

Rumbold A \& Crowther CA 2005b Vitamin E supplementation in pregnancy. Cochrane Database of Systematic Reviews. CD004069. (doi:10.1002/14651858.CD004069.pub2)

Scott N, Hodyl N, Osei-Kumah A, Stark M, Smith R \& Clifton V 2011 The presence of maternal asthma during pregnancy suppresses the placental pro-inflammatory response to an immune challenge in-vitro. Placenta 32 454-461. (doi:10.1016/j.placenta.2011.03.004)

Shah MD \& Shah SR 2009 Nutrient deficiencies in the premature infant. Pediatric Clinics of North America 56 1069-1083. (doi:10.1016/j.pcl. 2009.08.001)

Shoji H, Franke C, Campoy C, Rivero M, Demmelmair H \& Koletzko B 2006 Effect of docosahexaenoic acid and eicosapentaenoic acid supplementation on oxidative stress levels during pregnancy. Free Radical Research 40 379-384. (doi:10.1080/10715760500539147)

Shoji H, Franke C, Demmelmair H \& Koletzko B 2009 Effect of docosahexanoic acid on oxidative stress in placental trophoblast cells. Early Human Development 85 433-437. (doi:10.1016/j.earlhumdev. 2009.02.003)

Smuts CM, Huang M, Mundy D, Plasse T, Major S \& Carlson SE 2003 A randomized trial of docosahexaenoic acid supplementation during the third trimester of pregnancy. Obstetrics and Gynecology 101 469-479. (doi:10.1016/S0029-7844(02)02585-1)

Szajewska H, Horvath A \& Koletzko B 2006 Effect of n-3 long-chain polyunsaturated fatty acid supplementation of women with low-risk pregnancies on pregnancy outcomes and growth measures at birth: a meta-analysis of randomized controlled trials. American Journal of Clinical Nutrition 83 1337-1344.

Takahashi M, Tsuboyama-Kasaoka N, Nakatani T, Ishii M, Tsutsumi S, Aburatani H \& Ezaki O 2002 Fish oil feeding alters liver gene expressions to defend against PPAR $\alpha$ activation and ROS production. American Journal of Physiology. Gastrointestinal and Liver Physiology 282 G338-G348. (doi:10.1152/ajpgi.00376.2001)

Turner MA, Roulstone CJ, Desforges M, Cretney M, Champion E, Lacey H \& Greenwood SL 2006 The extent and variability of effects of culture conditions on the secretion of human chorionic gonadotrophin and interleukin- 6 by human, term placental explants in culture. Placenta $\mathbf{2 7}$ 98-102. (doi:10.1016/j.placenta.2004.12.004)

Weldon SM, Mullen AC, Loscher CE, Hurley LA \& Roche HM 2007 Docosahexaenoic acid induces an anti-inflammatory profile in lipopolysaccharide-stimulated human THP-1 macrophages more effectively than eicosapentaenoic acid. Journal of Nutritional Biochemistry 18 250-258. (doi:10.1016/j.jnutbio.2006.04.003)

Wong SW, Kwon MJ, Choi AM, Kim HP, Nakahira K \& Hwang DH 2009 Fatty acids modulate toll-like receptor 4 activation through regulation of receptor dimerization and recruitment into lipid rafts in a reactive oxygen species-dependent manner. Journal of Biological Chemistry 284 27384-27392. (doi:10.1074/jbc.M109.044065)

Received 2 June 2013

First decision 13 June 2013

Accepted 26 June 2013 